\title{
License Plate Segmentation Using Connected Component Analysis
}

\author{
${ }^{1}$ V.Karthikeyan, ${ }^{2}$ V.J.Vijayalakshmi, ${ }^{3}$ P.Jeyakumar \\ ${ }^{I}$ Assist Prof, Department of ECE, SVS College of Engineering, India \\ ${ }^{2}$ Assist Prof, Department of EEE, SKCET, India \\ ${ }^{3}$ Student Department of ECE Karpagam University, India
}

\begin{abstract}
Vehicle License Plate recognition has turned out to be a vital appliance of Intelligent Transportation Systems (ITS). In view of the fact that license plates can be put back, wrap or simply alter, they are not the eventual result for vehicle identification. The intention is to widen a structure where by Vehicle Identification Number (VIN) is digitally take picture and then identified by segmenting the characters from the images confined. In the proposed work a novel moves toward for segmentation of characters of license plate using Connected Component Analysis (CCA). In the beginning stage the identification number of the Vehicle was kept back in focal point and images were captured. The images were then focus to pre-processing which consists of image processing algorithms. These images were further powerfully developed by taking into account making the distance transform. The forgotten time and entropy results give a study for rising the effectiveness and highquality performance of CCA segmentation. The projected work presents a new method for License Plate Characters' Segmentation. The proposed approach is more effective than some of the presented method reported past.
\end{abstract}

Key Words: ITS- Intelligent Transportation Systems, Connected Component Analysis, Plate detection, Plate Cropping, Character Segmentation Normalization and Quantization.

\section{Introduction}

LPR (License Plate Recognition) is an image-processing technology used to identify vehicles by their license plates. In the recent years the number of vehicles is incessantly greater than before, the traffic conditions is worsening, which get enormous pressure to the world and atmosphere. Intelligent transport system is a realtime, accurate, and efficient transportation management system. This system can solve the various problems generated by the traffic obstruction. The vehicle license plate recognition system focuses mainly on the climatic conditions type of plate, alphabet etc. This projected approach is a new method for license plate identification from car images. CCA is a significant technique that examined and makes the pixels of a binarized image into components based on pixel connectivity. Each pixel is labeled with a value depending on the module to which it was allocated. The connected components are then examined to filter out extended and large components. Threshold of its spontaneous properties and simplicity of implementation, image thresholding enjoys an innermost position in application of image segmentation. Thresholding in a more prescribed way and lengthen it to techniques that are considerably more common. In the proposed work, For segmenting the Alphabets and the number from the license plate we carry out the steps they are plate cropping, plate normalization and plate quantization. The constructing fundamentals of dilations are pixel horizontal or vertical lines. Due to digits and characters, a license plate contains many vertical edges. This feature is employed for locating the plate in an image. Many approaches have been proposed for edge detection. Sobel mask has a high-quality presentation evaluated with other types; certainly, it is quick and easy. In general, there are two masks for Sobel, horizontal mask and vertical one. Closing also tends to smooth sections of contours but, as opposed to opening, it generally fuses narrow breaks and long thin gulfs, eliminates small holes, and fills gaps in the contour.

\section{Literature Review}

The first step is identifying number plate candidates from the vehicle; the proposed work starts with locating the number plate in the image. It is the toughest task in plate recognition the idea of this part is to identify probable candidate regions of the image in which the number plate might be contained. The next step is Region growing in which the characteristics of the number plate are identified such as shade and then readthrough each pixel in the image for the recognized uniqueness. If a pixel is identified as containing the characteristics of a number plate pixel all its adjacent pixels are checked to see if they hold the individuality of a number plate pixel. If a adjoining pixel holding the accurate uniqueness then we say that both pixels be in the right place to the similar region. This entire idea is recursively carried out awaiting each pixel in the image has been observed. Region growing is a speedy algorithm since each pixel is only observed once. Other advantages are that it extracts candidates with the correct shape, as it does not depend on the size of the region. Using the 
second method explained above to make the identified regions be the similar measurement or outline of a number plate makes region growing opposed to noise. A disadvantage of region growing is that since it is a recursive algorithm it requires a lot of memory usage. Setting the correct threshold for the preprocessing can also cause problems too when trying to create the binary image.

Extraction is the next step it is used to make simpler the character recognition stage, the license plate must first be extracted. There are several moves toward that can be in use to take out the font, Region splitting is used to separate element of an image into regions of parallel type. This can be practical to the resulting number plate that was extracted from the image. Region splitting identifies max out in a grey level histogram and then looks between the peaks for probable threshold values. Regions are identified as containing grey levels stuck between the thresholds. Region growing make use of morphology functions to search for a connected component (i.e. a character). The most excellent outcome accomplished using this method is outputted using a binary image-The extracted number plate is initially searched for a black pixel. When a black pixel is originated, it is understood that it is part of a character.

When a connected component is created it is then experienced to notice if it approaches the necessities of a character (e.g. size). This entire method is agreed out again and again until all the characters in the number plate have been extracted. This technique is extremely accurate in outcome the limits. Morphological operation consists of alteration that merge two sets by using vector addition of set SEs, and Erosion can be get hold of by dilating the complement of the black pixels and the enchanting the complement of the resultant point set. In erosion, each entity pixel that is touching a background pixel is altered into a background pixel. It makes the objects lesser. By subtracting the original image from the eroded image, the number plate would be extracted as there is a huge dissimilarity among the two images. Closing is the grouping of dilation-erosion with the identical SE. This operation can take away black holes on white objects. Opening is the grouping of erosion-dilation with the same SE. This operation can remove white holes on dark objects. Thus by this method we get an exact outcome. Neural Network-based filters and a post processor [12] in this paper it brings out the combination of two filtered images in regulate to place the license plates, the two Neural Networks used are vertical and horizontal filters, which inspect small windows of vertical and horizontal cross sections of an image and make a decision whether each window contains a license plate. Cross-sections have enough information for individual, a plate from the background. Hough transform [11] in this they have done the extraction of the license plate, the algorithm behind the technique consists of five steps. The horizontal and vertical line segments are combined and any rectangular regions identical the extent of a license plate is kept as candidate region. The disadvantage is that, this method requires huge memory and is computationally expensive. Detection Adaptive Gaussian filtering algorithm [5] their method is based on adapting the variance of the Gaussian filter to the noise characteristics and the restricted difference of the image data. Based on observations of how the human eye perceives edges in different images, they concluded that in areas with sharp edges, the filter variance should be small to preserve the sharp edges and keep the distortion small. In smooth areas, the variance should be greater, so as to filter out noise. The major drawback of this algorithm is that it assumes the noise is Gaussian with known variance.

In practical situations; however, the noise variance has to be estimated. The algorithm is also very computationally intensive .Wavelet Based Methods [4] as it was mentioned; analyzing an image at dissimilar scales increases the exactness and consistency of edge detection. Focusing on confined to a small area signal structures, e.g., edges, with a zooming procedure enables, simultaneous analysis from a rough to a fine shape. Progressing among scales also simplifies the inequity of edges versus textures. Because of having this skill, wavelet transform is an advantageous option for edge detection in different applications. Wavelet-based multiresolution expansions offer solid representations of images with regions of small difference alienated by highcontrast edges. Additionally, the use of wavelets provides a way to guess difference value for edges on a spacevarying basis in a limited or worldwide style as desirable. It consequences in coefficients that are purpose of the scale and shift. Therefore, WT acts as a geometric microscope, in which one can verify unlike parts of an image by just adjusting focal point on scale. A significant asset of WT is its capability to spotlight on localized structures, e.g. edges, with a zooming process that progressively reduces the scale stricture. In this way, coarse and fine signal structures are simultaneously analyzed at different scales.

\section{Related Work}

Adopting high-resolution sources is regarded as a trend in this territory, yet which also accompanies the shortcoming of long processing time. To cope with this, the Symmetric Mask-based Discrete Wavelet Transform (SMDWT) is employed to reduce the frame size without losing too much high frequency energy. It should be noted, regarding the memory requirements, that the SMDWT algorithm requires twice the image size space to perform the four mask filtering. In addition, the Hierarchical Ad boost (HA) is proposed to detect LPs in various situations. This method requires much less training time to achieve similar accuracy as that of the former Haar-like feature. In addition, this scheme can provide superior detection efficiency than that of the 
Haar-like feature. It is used for detection purpose but the disadvantage is that it will detect image with high false-positive rate. A projection-based Character Segmentation (CS) method is also proposed in this work, and which further considers the relationships among characters to improve the segmentation rate. The main drawback is that components in the middle are not recognized properly. The Naïve Bayes classifier with in total 13 different features is adopted for enhancing the recognition rate. Its main drawback is coding complexity. It has slow recognition rate. Its main disadvantage is that it can't learn interactions between features. In Binarization a License Plate usually contains two different colors to distinguish each of the characters from the background. However, the unpredictable lighting conditions and a dirt LP make it hard to separate the characters. A normal way is to use a fixed threshold for Binarization, yet it is rather coarse since the entire brightness of a LP suffered from the two above issues is not in balanced.

\section{Character Segmentation}

Figure illustrates the aspect definitions of a LP for improved understanding.

Initially, the top and bottom boundaries of the characters are established, and the horizontal projection is intended as below,

$$
\text { h_proji }=\sum_{j=1}^{1 v p} b_{i, j x}
$$

Where $\mathrm{Wp}$ indicates the thickness of an extracted LP, and $\mathrm{h} \_$proje $\mathrm{i}_{\mathrm{i}}$ stands for the outcome subsequent to horizontal projection. Figure shows an example, and which is obtained from the B in Fig. 3.1.2.1(a). According to the observation, the gray circles in Fig. 3.1.3.2(a) indicate the majority probable top (the right circle) and bottom (the left circle) boundaries of the LP. Yet, there are some other negligible projection points which may get in the way the detection precision.

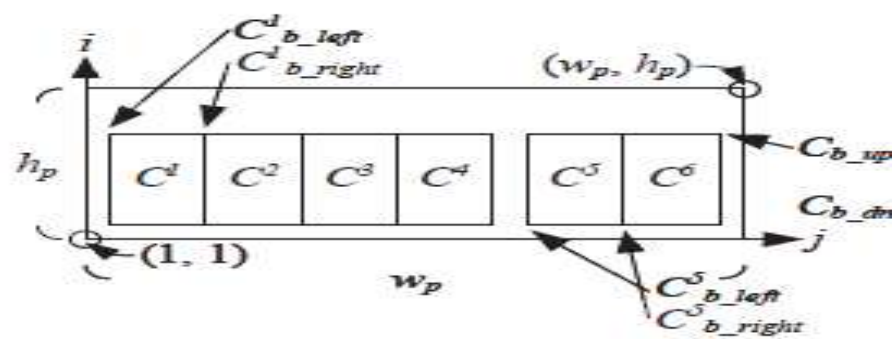

Fig (a) Aspect definitions of LP

To handle with this, the searching approach is given below,

$$
\begin{aligned}
& \mathrm{hp}\left(h_{\mathrm{ip},}, i\right)=\mathrm{h} \_p r o j_{\mathrm{i}}+\mathrm{h} \_ \text {proj } j_{i+h i p} \text {, } \\
& \mathbb{H}=\operatorname{argmin}_{(\mathrm{hip}, \mathrm{i})} \mathrm{hp}\left(\mathrm{h}_{\mathrm{ip}, \mathrm{i}} \mathrm{i}\right) \text {, where } \mathbb{H}=\left\{\left(\mathrm{h}_{\mathrm{ip}, \mathrm{i}} \mathrm{i}\right)\right\} \text {, }
\end{aligned}
$$

where $\mathrm{h}_{\mathrm{ip}}$ and $\mathrm{i}$ represent the imaginary height of the LP and vertical coordinate of LP, respectively; $\mathrm{H}$ denotes the set of the combination of $\left(\mathrm{h}_{\mathrm{ip}} \mathrm{i}\right)$ which presents the minimum $\mathrm{hp}\left(\mathrm{h}_{\mathrm{ip}}, \mathrm{i}\right)$. Normally $\mathrm{H}$ does not offer a exceptional solution $(|H|>1)$, and thus some constrains have to be recognized. The initial is the bottom boundaries of LPs should place inside the vertical region $1 \leq \mathrm{i} \leq \mathrm{p} / 2$; the top boundaries of LPs should position within the vertical region/2 $\leq \mathrm{i} \leq \mathrm{p}$. The second limit is formulated as below,

$$
\left(\mathrm{h}_{\mathrm{ip}, \mathrm{opt}}, \mathrm{i}_{\mathrm{opt}}\right)=\min _{\text {hip }}\left\{\left(\mathrm{h}_{\mathrm{ip}}, \mathrm{i}\right) \mid\left(\mathrm{h}_{\mathrm{ip}}, \mathrm{i}\right) \square \mathrm{sH}\right\}
$$

where ${ }_{s}$ which meets the first constrain. Subsequently, the bottom and top boundaries can be easily resulting by $\mathrm{C}_{\mathrm{b}_{-} \mathrm{dn}}=\mathrm{i}_{\mathrm{opt}}$ and $\mathrm{Cb}_{-}$up $=\mathrm{i}_{\text {opt }}+\mathrm{h}_{\mathrm{ip}, \text { opt }}$ respectively.

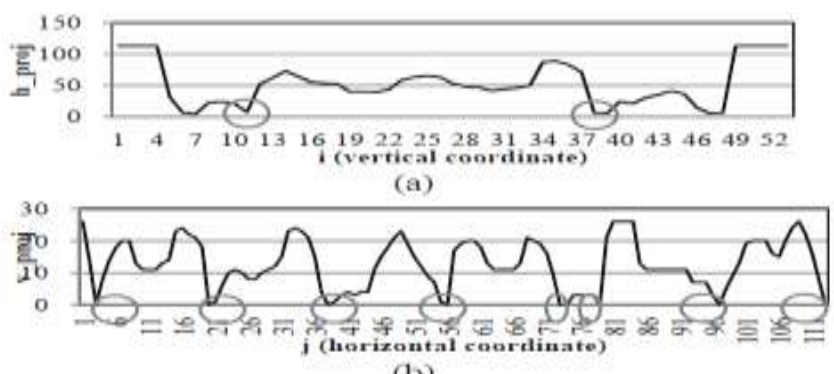

(b)

Fig 3 relative projection results (a) Horizontal and (b) vertical projection results

The left and right boundaries of every nature are resultant with the identical way as above. Notably, the utilized binary result for the following vertical projection of a LP is recalculated with the mode introduced where binarization vertical range is limited within $\left[\mathrm{C}_{\mathrm{b}_{-} \mathrm{dn}} \mathrm{C}_{\mathrm{b}_{-u p}}\right]$. The corresponding results are shown in Fig. 
3.1.2.1(f)andthe horizontal projection of $\mathrm{LP}$ is calculated as

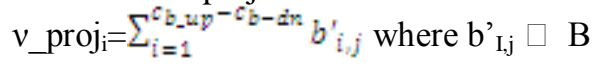

Particularly, the probable calculated choice of the vertical projection is restricted inside the region of the characters from a common complete vehicle plate because of the affects from other non-character parts. It is noteworthy that there are two possible arrangements of the LPs in Taiwan, for example TR-5894 and 6675-WU, named type 1 (t1) and type 2 (t2), respectively, in this paper. The notation "-" is used to enhance the positions in separating the two groups, numbers and characters. Thus, the CS algorithm has to be modified by considering this characteristic. As eight points with gray circles have the minimum magnitude of projections, and the corresponding calculation is arranged as below.

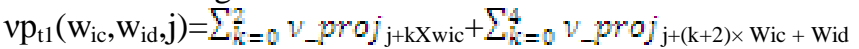

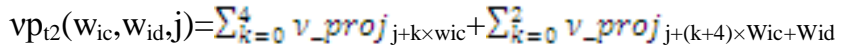

$$
\begin{aligned}
& \left(\mathrm{W}_{\mathrm{ic}, \mathrm{opt}}, \mathrm{W}_{\mathrm{id}, \mathrm{opt}}, \mathrm{j}_{\mathrm{opt}}\right)=\min \left\{\operatorname{argmin} v \mathrm{p}_{\mathrm{t} 1}\left(\mathrm{w}_{\mathrm{ic}}, \mathrm{W}_{\mathrm{id}}, \mathrm{j}\right)\right. \text {, } \\
& \left.\left(\mathrm{w}_{\mathrm{ic}}, \mathrm{w}_{\mathrm{id}}, \mathrm{j}\right) \quad \operatorname{argmin}_{\left(\mathrm{Wic}_{\mathrm{ic}}, \mathrm{Wid}_{\mathrm{j}}\right)} \mathrm{vp}_{\mathrm{t} 2}\left(\mathrm{w}_{\mathrm{ic}}, \mathrm{W}_{\mathrm{id}} \mathrm{j}\right)\right\} \text {, }
\end{aligned}
$$

Where $\mathrm{W}_{\mathrm{ic}}$ and $\mathrm{W}_{\mathrm{id}}$ denote the imaginary width of a character and the imaginary width of the "-" notation in Taiwan's LPs, respectively, and is defined at $\mathrm{w}_{\mathrm{ic}} / 4 ; \mathrm{j}$ denotes the horizontal coordination of a LP. According to the calculation, the boundaries of

$$
\begin{aligned}
& C_{\text {Bleft }}^{k}=\left\{\begin{array}{l}
\text { jopt }+ \text { Wic, opt } \times(k-1), \text { if } 1 \leq \mathbb{k} \leq \text { ncg } \\
\text { jopt }+ \text { Wic opt } \times(k-1)+W_{i d} \text { opt }_{*} \text { O. W. }
\end{array}\right.
\end{aligned}
$$

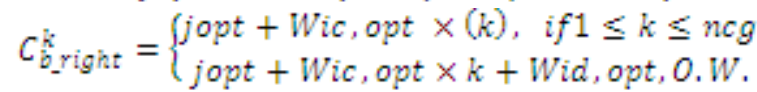

$$
\begin{aligned}
& \mathrm{ncg}=\left\{\begin{array}{l}
2, \text { if the used }\left(w_{\text {ie, opt }}, w_{\text {id opt }} j_{\text {opt }}\right) \text { belongs to } t 1 \\
4, \text { if the used }\left(w_{\text {ie opts }} w_{\text {id opt }} j_{\text {opt }}\right) \text { belongs to } t 2
\end{array}\right.
\end{aligned}
$$

Characters $\mathrm{C}_{{ }_{\mathrm{b} \_ \text {left }}}^{\mathrm{k}}$ and $\mathrm{C}_{\mathrm{b}_{\text {_right }}}$ and can be segmented by $\left(\mathrm{W}_{\mathrm{ic}, \mathrm{opt}}, \mathrm{W}_{\mathrm{id}, \mathrm{opt}}, \mathrm{j}_{\mathrm{opt}}\right)$ as where $\mathrm{k}$ indicates the kth character in LP in arrange from left to right; ncg is a patchy to denote dissimilar types of LPs. Finally, the boundary of each character can be determined by the above horizontal and vertical projections. According to these extracted boundaries, each character are cropped from the original grayscale basis and normalized to 10x20.These cropped grayscale basis's will be binarized again with its mean, The object with maximum area size from the binarized results gone through connecting component processing is employed for further processing.

\section{Character Segmentation Using Cca}

\section{Proposed Work}

Character segmentation is the process of extracting the characters and numbers from the license plate image. Miscellaneous features make the character segmentation mission difficult, like image noise, plate frame, space mark, plate's rotation and light variance. A number of actions have been proposed for character segmentation to overcome these problems. After removing the plate borders in the previous step, this step starts with removing the noise from the plate. The proposed work is used for character segmentation is based on thresholding and CCA.In binary image processing, CCA is an important technique that scans and labels the pixels of a binarized image into components based on pixel connectivity. Each pixel is labeled with a value depending on the component to which it was assigned. The connected components are then analyzed to filter out long and wide components and only left the components according to the defined values

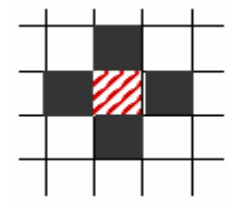

Fig 4-neighbourhood

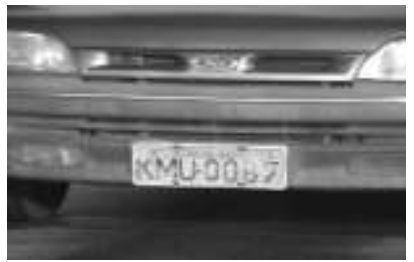

Fig.5 (A) Car images segmented license plates on right

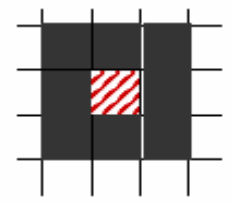

(2) 8-neighbourhood

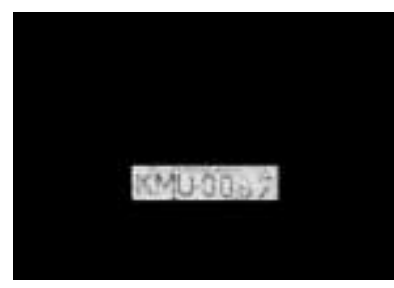




\section{License Plates Crop And Normalization}

Given the coordinate and dimension of specific object, we can crop it from image and its equivalent image. This object crop is obtained from gray image. Now we find the angle of this object with horizontal pivot and it rotates in opposite direction with same angle and converts to binary image. In image processing normalization is a process that changes the range of pixel intensity values. Applications include photographs with poor contrast due to glare, for example. Normalization is sometimes called contrast stretching. In more general fields of data processing, such as Digital Signal Processing, it is referred to as dynamic range The purpose of dynamic range expansion in the various applications is usually to bring the image, or other type of signal, into a range that is more familiar or normal to the senses, hence the term normalization. Often, the motivation is to achieve consistency in dynamic range for a set of data, signals, or images to avoid mental distraction or fatigue. For example, a newspaper will strive to make all of the images in an issue share a similar range of grayscale Quantization, involved in image processing, is a lossy compression technique achieved by compressing a range of values to a single quantum value. When the number of discrete symbols in a given stream is reduced, the stream becomes more compressible. For example, reducing the number of colors required to represent a digital image makes it possible to reduce its file size.
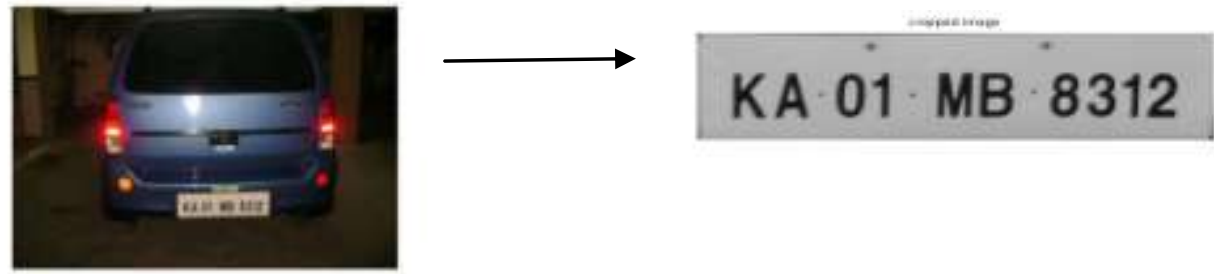

Fig.6 Cropped Image

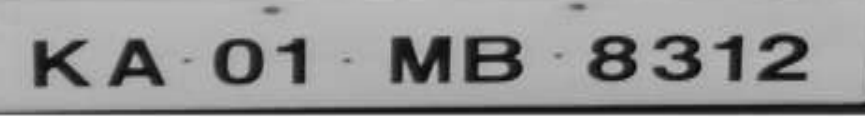

Fig.7 Normalized Plate Image

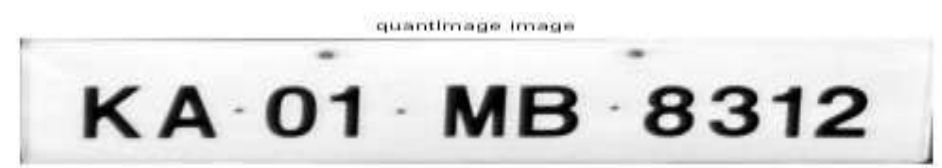

Fig.8 Quantized License Plate

\section{Conclusion}

The projected exertion obtains a description attempt of the segmentation of License Plate. The anticipated method describe that detection takes place in a efficient manner and Binary Thresholding method is used to separate object and background. Morphological methods were used to achieve the detection of License Plate. Plate detection and plate segmentation rate of the proposed work is so accurate and detection rate is very much prompt. The connected components are then analyzed to filter out long and wide components and only left the components according to the defined values. CCA convey imperative technique that scans and labels the pixels of a binarized image into components based on pixel connectivity Quantization, concerned in image processing, is a glossy compression technique achieved by compressing a range of values to a single quantum value. When the number of distinct symbols in a given stream is reduced, the brook becomes more compressible. As a result Vehicle plate segmentation from the vehicle engages in recreation is trouble-free and effectual task in recognizing the License plate. In prospect the researchers are so eminent to grip certain new attitude in recognizing the License plate.

\section{Experimental Results}

In our anticipated work the experimental results make use of a necessary task Fig. 8 demonstrate the Original Image after Localization, Fig.9 illustrates the Cropped image from the detected License plate from the 
vehicle where the plate going to be segmented. Fig.10 Shows the quantized image, which is a glossy compression technique achieved by compression Fig. 11 shows the segmented Normalized image.

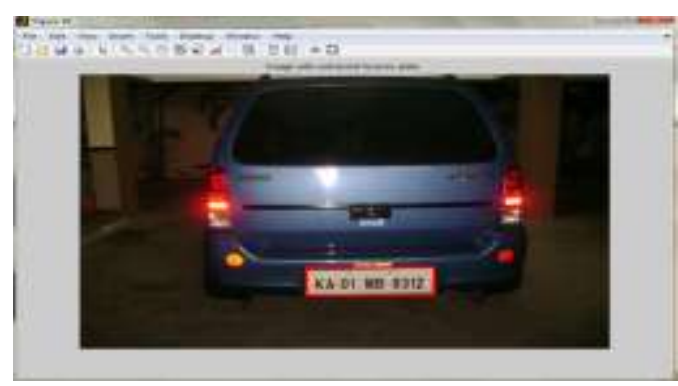

Fig.8 Original Image after Localization

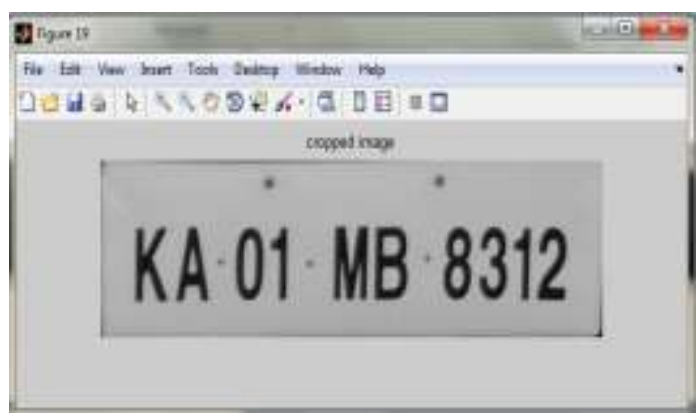

Fig.9 Cropped Image

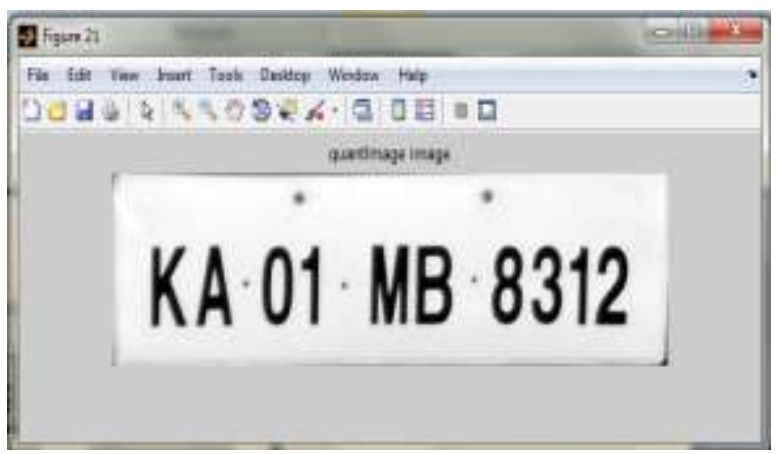

Fig.10 Quantization image

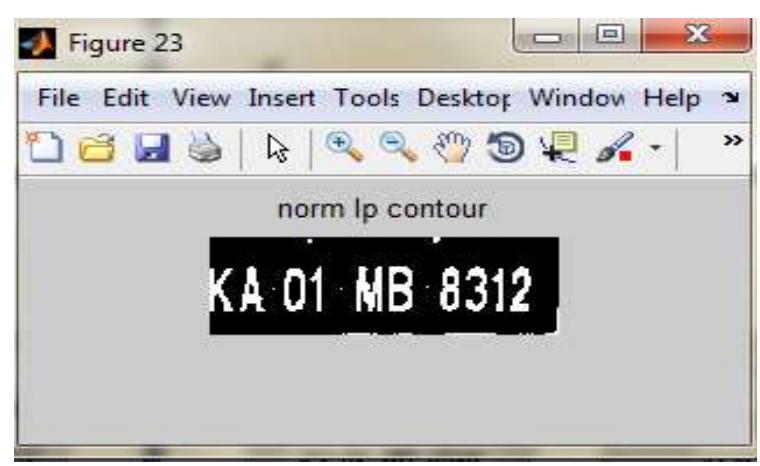

Fig.11 Normalization Image 


\section{References}

[1] Adebayo daramola1, e. adetiba1, a. u. adoghe1, j. a. badejo1, i. a samuel1 and t. fagorus (2011), on 'automaticvehicle identification system using license plate '.

[2] Comelli, P., Ferragina, P., Granieri. M. N., and Stabile, F.(1995),on 'Optical recognition of motor vehicle license plates' , IEEE Transactions on Vehicular Technology, vol. 44, no. 4, pp: 790-799,

[3] Cowell, J., and Hussain, F.(2002), on 'A fast recognition system for isolated Arabic characters', Proceedings Sixth International Conference on Information and Visualisation, IEEE Computer Society, London, England, pp. 650-654, 2002.

[4] Cowell, J., and Hussain, F.,(2001),on 'Extracting features from Arabic characters',Proceedings of the IASTED International Conference on COMPUTER GRAPHICS AND IMAGING, Honolulu, Hawaii, USA, pp. 201-206, 2001.

[5] G. Deng and L.W. Cahill,(1994), on 'An adaptive Gaussian filter for noise reduction and edge detection', $\square$ in Proc. IEEE Nucl. Sci. Symp. Med. Im. Conf., 1994, pp. 1615-1619.

[6] Hansen, H., Kristensen, A. W., Kohler, M. P., Mikkelsen, A. W. , Pedersen J. M., and Trangeled, M.,(2002), on 'Automatic recognition of license plates', Institute for Electronic System, Aalhorg University, May 2002.

[7] Hontani, H., and Koga, T.,(2001),on 'Character extraction method without prior knowledge on size and information', Proceeding s of the IEEE International Vehicle Electronics Conference (IVEC'01), pp. 67-72, 2001.

[8] Hu, M. K., (1962) on 'Visual Pattern Recognition by Moment Invariant', IRE Transaction on Information Theory, vol IT - 8, pp. $179-187$.

[9] Jing-Ming Guo, Senior Member, IEEE, Yun-Fu Liu, Student Member, IEEE, and Chih-Hsien Hsia, Member, IEEE ,2012, on Multiple License Plates Recognition System'.

[10] Khalid Maglad, Dzulkifli Mohamad, Nureddin A. Abulgasem (2011), on 'Saudian Car License Plate Number Detection and Recognition Using Morphological Operation and RBF Neural Network'.

[11] Kim, G. M., (1997), on 'The automatic recognition of the plate of vehicle using the correlation coefficient and Hough transform', Journal of Control, Automation and System Engineering, vol. 3, no.5, pp. 511-519, 1997. 75 .

[12] Kim, K. K., Kim, K. I., Kim, J.B., and Kim, H. J.(2000), on 'Learning based approach for license plate recognition', Proceedings of IEEE Processing Society Workshop on Neural Networks for Signal Processing, vol. 2, pp: 614-623, 2000.

[13] S.Kranthi, K.Pranathi, A.Srisaila, (July 2011) on' Automatic Number Plate Recognition'.

[14] KTATA, Faouzi BENZARTI and Hamid AMIRI(2012), on 'Detection Using Mathematical Morphology'.

[15] Lee, E. R., Earn, P. K., and Kim, H. J. (1994), on Automatic recognition of a car license plate using color image processing', IEEE International Conference on Image Processing 1994, vol. 2, pp.301-305.

[16] Mitra Basu, -Gaussian-Based Edge-Detection Methods(2002), on 'A Survey $\square$, IEEE Transactions On Systems, Man, And Cybernetics', Vol. 32, No. 3, August 2002, pp. 252-260 . 